\title{
ZUM NEUAUFBAU DER STAATSANWALTSCHAFT IN DER VOLKSREPUBLIK CHINA
}

\author{
WolfGang KessLeR
}

Nach dem Tod des Partei- und Staatsführers Mao Zedong und dem Sturz der ,,Viererbande“ hat in der Volksrepublik China erneut eine Verrechtlichung des Staatslebens begonnen. Nach einer neuen, detaillierten Verfassung, die mehr der ersten volksrepublikanischen Verfassung von 1954 ähnelt und ihre vage programmatische Vorgängerin von 1975 ablöst $^{1}$, wurden im Sommer 1979 vom Nationalen Volkskongreß weitere Gesetze zur Neuorganisierung des Staats- und Wirtschaftslebens verabschiedet. Das labile Gleichgewicht zwischen Gesetzmäßigkeit der Verwaltung und Unparteilichkeit der Strafverfolgung einerseits und der beherrschenden Stellung, die andererseits die Kommunistische Partei innehat, gestaltete die Arbeit einer Staatsanwaltschaft, die keinen äußeren Einflüssen unterworfen sein sollte ${ }^{2}$, stets sehr schwierig, ermöglichte zahlreiche Formen irregulärer Einflußnahme und schließlich auch die faktische Beseitigung aller nach festen Regeln funktionierenden Justiz- und Strafverfolgungsbehörden im Zug der „,Großen Proletarischen Kulturrevolution“.

Nachdem die ,Viererbande“ im Herbst 1976 aus der Macht gedrängt worden war und die Tendenz zur Juridifizierung wieder erstarkte, begann auch die offizielle chinesische Presse eine breite Diskussion über die Reform und den Neuaufbau des Rechtswesens ${ }^{3}$. In der allgemeinen Forderung, wieder zu verfaßter Herrschaft zurückzukehren, galt die Aufmerksamkeit vieler - und zuweilen prominenter ${ }^{4}$ - Autoren auch dem Wiederaufbau einer unbeeinflußten, nach dem Legalitätsgrundsatz operierenden Staatsanwaltschaft. Das hier vorzustellende Gesetz über die Organisation der Volksstaatsanwaltschaft der Volksrepublik China vom 1. Juli 1979 löst das erste volkschinesische Gesetz von 1954 über die Volksstaatsanwaltschaft ab. Die chinesische Gesetzgebung während der Anfangsphase der Volksrepublik lehnte sich eng an die Sowjetunion an, und auch die Volksstaatsanwaltschaft wurde damals nach deren Vorbild konzipiert. Dementsprechend war die Volksstaatsanwaltschaft nach dem 1954er Gesetz nicht nur Strafverfolgungebehörde und Aufsichtsbehörde für die Strafvollstreckung, sondern ein Organ zur umfassenden Uberwachung der allgemeinen Rechtmäßigkeit sowohl staatlichen Verwaltungshandelns als auch des Verhaltens der Bürger $^{5}$. Zu diesem Zweck war die Volksstaatsanwaltschaft neben ihren Aufsichts- ${ }^{6}$, Prüfungs- ${ }^{7}$ und Beschwerderechten ${ }^{8}$ bezüglich Behörden und Unternehmen auch befugt, in ,für den Staat oder das Volk bedeutenden Zivilsachen" Prozesse zu beginnen oder an ihnen teilzunehmen ${ }^{9}$. Das neue Gesetz von 1979 bringt eine Abkehr von der Staatsanwaltschaft als Behörde zu umfassender Rechtsüberwachung und beschränkt ihre Aufgaben auf die Strafverfolgung, das gerichtliche Strafverfahren und die Strafvollstreckung ${ }^{10}$.

\footnotetext{
1 Text der Verfassungen von 1975 und 1978 in: Verfassungstexte (Beilage zu VRU 3/1977 bzw. 3/1978).

$2 \S 6$ Gesetz von 1954

3 Vergleiche die Aufsätze zum Strafrecht in: Huang/Keßler/Liu/Münzel, Recht in China, Aufsätze aus der Volksrepublik China zu Grundsatzfragen des Rechts, Mitteilungen des Instituts für Asienkunde Hamburg, No. 104, Hamburg 1979.

4 Etwa des Leitenden Obersten Volksstaatsanwalts Huang Huoqing in Recht in China, S. $101 \mathrm{ff}$.

5 \4 Gesetz von 1954.

$6 \$ 4$ Gesetz von 1954

$7 \$ 8$ Gesetz von 1954

$8 § 8$ Gesetz von 1954.

94 No. 6 Gesetz von 1954

$10 \iint 1,5$ Gesetz von 1979.
} 
Die neuen Bestimmungen über die Aufgaben der Volksstaatsanwaltschaft im Bereich des Strafrechts gleichen im wesentlichen denen von 1954, sind aber in einigen Punkten präzisiert und erweitert. In der Vergangenheit ließen oft endlos schwebende Ermittlungsverfahren Beschuldigte im Ruch der Kriminalität, obwohl bei einer Entscheidung der Volksstaatsanwaltschaft über die Klageerhebung das Verfahren hätte eingestellt werden müssen. Daher rührende Stigmatisierung Unschuldiger wurde häufig in der Presse beklagt. Das neue Gesetz schreibt nun, neben der Statuierung des herkömmlichen Legalitätsgr!'ndsatzes für die Strafverfolgung, weitergehend ausdrücklich vor, daß ein Ermittlungsverfahren einzustellen ist, wenn die Ermittlungen die Klageerhebung nicht rechtfertigen ${ }^{11}$. Ebenfalls präzisiert sind die Bestimmungen über das polizeiliche Klageerzwingungsverfahren ${ }^{\mathbf{1 2}}$ und die Freiheit der Volksstaatsanwaltschaft von äußerer Einflußnahme, die sich nun neben Staatsorganen auch auf ,,Verbände und Einzelpersonen “ bezieht ${ }^{13}$. Es bleibt abzuwarten, ob und wieweit die Schranke gegen ,,Verbände" auch auf die Kommunistische Partei angewendet werden wird; wahrscheinlich ist jedenfalls, daßdie Norm sich leichter gegen $z$. B. die Jugendliga oder die Frauenvereinigung richten lassen wird.

Organisatorisch ist die Volksstaatsanwaltschaft enger als 1954 mit den örtlichen Parlamenten verzahnt und gleichzeitig die hierarchische Leitung der Behörde durch Genehmigungsvorbehalte für die Ernennung von Staatsanwälten erhalten worden ${ }^{14}$ : Ernennungen erfolgen durch das Parlament der jeweiligen Ebene mit zusätzlicher Genehmigung, die der nächsthöhere Leitende Volksstaatsanwalt vom Ständigen Ausschuß des Parlaments dieser nächsthöheren Ebene einzuholen hat ${ }^{15}$. Die Staatsanwaltschaften aller Ebenen sind Parlamenten derselben Ebene verantwortlich und berichtspflichtig16.

Das neue Gesetz über die Volksstaatsanwaltschaft bemüht sich, wie die anderen neuen Gesetze zum materiellen und formellen Strafrecht ${ }^{17}$, seine Materie in Einzelheiten und berechenbar zu regeln, und es ist zu erwarten, daß diese Reform im Rahmen der Staatsordnung der Volksrepublik China helfen wird, die spezifisch normlosen Exzesse der , Kulturrevolution" zu beenden.

Für Erläuterungen der Namen geographischer Verwaltungseinheiten sei der Leser verwiesen auf: Martin/Liao, Chinesisch-deutscher Wortschatz, Politik und Wirtschaft der VR China, Berlin etc.: Langenscheidt 1977; ,,Zizhi-zhou“ (bei Martin/Liao ,,autonome Region“) ist hier als ,,autonomer Bezirk“ übersetzt.

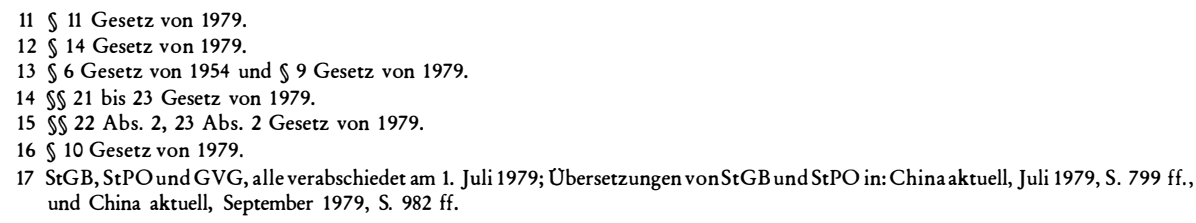




\section{GESETZ UBER DIE ORGANISATION \\ DER VOLKSSTAATSANWALTSCHAFT DER VOLKSREPUBLIK CHINA}

(Beschlossen auf der Fünften Vollsitzung der Zweiten Tagung des Fünften Nationalen Volkskongresses am 1. Juli 1979)

\section{ERSTES KAPITEL}

Allgemeine Regeln

\section{$\int 1$}

Die Volksstaatsanwaltschaft der Volksrepublik China ist ein staatliches Organ zur Rechtsüberwachung.

$\int 2$

Die Volksrepublik China errichtet die Oberste Volksstaatsanwaltschaft, örtliche Volksstaatsanwaltschaften aller Ebenen sowie Sondervolksstaatsanwaltschaften.

Die örtlichen Volksstaatsanwaltschaften aller Ebenen gliedern sich in:

(1) Volksstaatsanwaltschaften der Provinzen, Autonomen Gebiete und regierungsunmittelbaren Städte;

(2) Abteilungsvolksstaatsanwaltschaften der Provinzen, Autonomen Gebiete und regierungsunmittelbaren Städte und Volksstaatsanwaltschaften der Autonomen Bezirke und provinzunmittelbaren Städte;

(3) Volksstaatsanwaltschaften der Kreise, Städte, Autonomen Kreise und stadtunmittelbaren Gebiete.

Die Volksstaatsanwaltschaften der Provinz- und Kreisebenen können gemäß (ihren) Arbeitserfordernissen und nach Genehmigung des Ständigen Ausschusses der Volksvertreterversammlung derselben (jeweiligen) Ebene in Bergwerksgebieten, landwirtschaftlichen Erschließungsgebieten und Forstgebieten Volksstaatsanwaltschaften in Form entsandter Behörden einrichten.

Die Sondervolksstaatsanwaltschaften schließen ein: Militärstaatsanwaltschaften, Eisenbahntransportstaatsanwaltschaften, Wassertransportstaatsanwaltschaften und andere Sonderstaatsanwaltschaften.

Die Einrichtung der Sondervolksstaatsanwaltschaften, ihr Aufbau und ihre Befugnisse werden vom Ständigen Ausschuß des Nationalen Volkskongresses anderweitig geregelt.

$\int 3$

Die Volksstaatsanwaltschaften aller Ebenen bestellen (jeweils) einen Leitenden Staatsanwalt und mehrere Stellvertretende Leitende Staatsanwälte und Staatsanwälte. Der Leitende Staatsanwalt leitet einheitlich die Arbeit der (jeweiligen) Staatsanwaltschaft.

Die Volksstaatsanwaltschaften aller Ebenen richten Staatsanwaltsausschüsse ein. Die Staatsanwaltsausschüsse praktizieren das System des demokratischen Zentralismus und erörtern und entscheiden unter der Anleitung des Leitenden Staatsanwalts bedeutsame Fälle und andere bedeutsame Fragen. Wenn ein Leitender Staatsanwalt in bedeutsamen Fragen einer Mehrheitsentscheidung nicht zustimmt, kann um Entscheidung durch den Ständigen Ausschuß der Volksvertreterversammlung derselben Ebene nachgesucht werden. 
$\int 4$

Durch die Ausübung ihrer Ermittlungsrechte unterdrückt die Volksstaatsanwaltschaft alle landesverräterischen, staatsspalterischen und anderen konterrevolutionären Aktivitäten, bekämpft konterrevolutionäre und andere verbrecherische Elemente, wahrt die Einheit des Staates, das System der Diktatur des Proletariats, die sozialistische Rechtsordnung, die Ordnung (in) der Gesellschaft, der Produktion, der Arbeit, Erziehung und Wissenschaft und dem Leben der Volksmassen, schützt das im sozialistischen Eigentum des ganzen Volkes, das im kollektiven Eigentum der werktätigen Massen und das rechtmäßige im Privateigentum der Bürger stehende Vermögen sowie die Rechte der Person, die demokratischen Rechte und andere Rechte der Bürger und schützt den ungestörten Fortgang des Aufbaus der sozialistischen Modernisierung.

Durch ihre Ermittlungstätigkeit erzieht die Volksstaatsanwaltschaft die Bürger zur Treue zum sozialistischen Vaterland, zur Einhaltung von Verfassung und Gesetzen aus eigener Einsicht und zu aktivem Kampf gegen gesetzesverletzendes Verhalten.

$\llbracket 5$

Die Volksstaatsanwaltschaften aller Ebenen üben folgende Befugnisse aus:

(1) Ausübung der Ermittlungsrechte gegenüber Landesverrat und Staatsspaltung sowie bedeutenden Straffällen, in denen der einheitlichen Ausführung der staatlichen Richtlinien, Gesetze, Verordnungen und Erlasse ernstlicher Schaden zugefügt wurde;

(2) Durchführung der Aufklärung in direkt zur Bearbeitung übernommenen Straffällen;

(3) Prüfung von durch Polizeiorgane aufgeklärten Fällen und Entscheidung, ob zu verhaften oder Klage zu erheben oder von Klageerhebung abzusehen ist; Úberwachung, ob die Aufklärungstätigkeit der Polizeiorgane rechtmäßig ist;

(4) Erhebung der öffentlichen Klage in Straffällen und Vertretung der (so erhobenen) öffentlichen Klage; Ủberwachung, ob das Verfahren in den Volksgerichten gemäß den Gesetzen (durchgeführt) ist;

(5) Uberwachung der Gesetzmäßigkeit der Vollstreckung von Strafurteilen und Beschlüssen in Strafsachen sowie der Tätigkeit von Strafanstalten, Untersuchungshaftanstalten und Arbeitsumerziehungsanstalten.

$\int 6$

Die Volksstaatsanwaltschaft garantiert gemäß den Gesetzen das Recht der Bürger, Strafanzeige wegen Gesetzesverletzung durch Staatsbedienstete zu stellen und ermittelt die rechtliche Verantwortlichkeit von Personen, die Rechte der Person, demokratische Rechte oder andere Rechte der Bürger verletzt haben.

$\$ 7$

Die Volksstaatsanwaltschaft hat bei ihrer Arbeit die Wahrheit in den Tatsachen zu suchen, gründlich die Massenlinie durchzuführen, die Kritik der Massen anzuhören, sich der Uberwachung durch die Massen zu unterziehen, Nachforschungen anzustellen, das Schwergewicht auf Beweise zu legen und nicht leichtfertig mündlichen Geständnissen Glauben zu schenken, strikt zu unterbinden, daß erpreßten Geständnissen geglaubt wird und konkret die Widersprüche zwischen uns und dem Feind und die Widersprüche im Volk zu unterscheiden und zu behandeln.

Die Bediensteten der Volksstaatsanwaltschaften aller Ebenenhaben getreu dem wahren Bild der Tatsachen, getreu dem Gesetz und getreu der sozialistischen Sache mit vollem Einsatz dem Volk zu dienen. 


\section{$\int 8$}

Die Volksstaatsanwaltschaften aller Ebenen haben in der Ausübung ihrer Ermittlungsrechte bei der Anwendung der Gesetze gegen alle Bürger durchweg gleich zu verfahren; es sind keinerlei Vorrechte zulässig.

\section{$\$ 9$}

Die Volksstaatsanwaltschaften üben den gesetzlichen Bestimmungen gemäß ihre Ermittlungsrechte unabhängig aus und unterliegen keinen Eingriffen durch andere Verwaltungsorgane, Verbände und Einzelpersonen.

\section{$\$ 10$}

Die Oberste Volksstaatsanwaltschaft ist in ihrer Arbeit dem Nationalen Volkskongreß und . dessen Ständigem Ausschuß verantwortlich und zum Bericht verpflichtet. Die örtlichen Volksstaatsanwaltschaften aller Ebenen sind in ihrer Arbeit den Volksvertreterversammlungen derselben (jeweiligen) Ebene und deren Ständigen Ausschüssen verantwortlich und zum Bericht verpflichtet.

Die Oberste Volksstaatsanwaltschaft leitet die Arbeit der Volksstaatsanwaltschaften aller Ebenen sowie der Sondervolksstaatsanwaltschaften; die Volksstaatsanwaltschaften der höheren Ebene leiten die Arbeit der Volksstaatsanwaltschaften der niederen Ebene(n).

\section{ZWEITES KAPITEL}

Verfahren zur Ausübung der Befugnisse der Volksstaatsanwaltschaft

\section{$\S 11$}

Wenn die Volksstaatsanwaltschaft entdeckt und überzeugt ist, daß eine Straftat begangen worden ist, hat sie gemäß dem Gesetz ein Verfahren einzuleiten oder (den Fall) den Polizeiorganen zur Aufklärung zu übergeben. Wenn nach Abschluß der Aufklärung die Volksstaatsanwaltschaft der Uberzeugung ist, daß die strafrechtliche Verantwortlichkeit des Beschuldigten ermittelt werden muß, hat sie beim Volksgericht die öffentliche Klage zu erheben; wenn sie der Uberzeugung ist, daß die strafrechtliche Verantwortlichkeit nicht ermittelt werden muß, hat sie das Verfahren einzustellen.

$\S 12$

Jede Verhaftung eines Bürgers, mit Ausnahme der von Volksgerichten angeordneten, bedarf der Genehmigung durch die Volksstaatsanwaltschaft.

$\S 13$

In Fällen, in denen die Polizeiorgane die Erhebung der öffentlichen Klage begehren, hat die Volksstaatsanwaltschaft eine Prüfung vorzunehmen und zu entscheiden, ob die Klage erhoben wird, von der Klageerhebung abgesehen oder die (Berechtigung der) Klageerhebung abgelehnt wird. Soweit wichtige Umstände der Straftat nicht klar oder Beweise unzureichend sind, kann (die Sache) zur ergänzenden Aufklärung an die Polizeiorgane zurückgegeben werden.

Wenn die Volksstaatsanwaltschaft entdeckt, daß die Aufklärungstätigkeit der Polizeiorgane gegen Gesetze verstößt, hat sie die Polizeiorgane anzuweisen, (die Verstöße) abzustellen. $§ 14$

In Fällen von durch die Polizeiorgane übersandten Sachen, in denen die Volksstaatsanwaltschaft eine Verhaftungsgenehmigung oder die Erhebung der öffentlichen Klage abgelehnt oder von der Klageerhebung abgesehen hat, können die Polizeiorgane, wenn sie (die Entscheidung) für falsch halten, einen Neubescheid der Volksstaatsanwaltschaft und ebenfalls 
eine Nachprüfung durch die Volksstaatsanwaltschaft der (nächst-)höheren Ebene verlangen. Die Volksstaatsanwaltschaft der (nächst-)höheren Ebene hat rechtzeitig eine Entscheidung zu treffen und die Volksstaatsanwaltschaft der niederen Ebene sowieso die Polizeiorgane anzuweisen, die Entscheidung auszuführen.

$\$ 15$

In Fällen, wo die Volksstaatsanwaltschaft die öffentliche Klage erhoben hat, übernimmt der (jeweilige) Leitende Staatsanwalt oder ein Staatsanwalt die Sitzungsvertretung als öffentlicher Ankläger, vertritt die (erhobene) öffentliche Klage und überwacht, ob das (gerichtliche) Verfahren dem Gesetz gemäß abläuft.

$\S 16$

In den Fällen, wo die Volksstaatsanwaltschaft Klage erhoben hat, kann das Volksgericht, wenn es der Uberzeugung ist, daß wichtige Umstände der Straftat nicht klar oder Beweise unzureichend sind oder daß Gesetze verletzt sind, (die Sache) zur ergänzenden Aufklärung an die Volksstaatsanwaltschaft zurückgeben oder diese anweisen, die Gesetzesverletzungen (im Ermittlungsverfahren) abzustellen.

$\$ 17$

Die örtlichen Volksstaatsanwaltschaften jeder Ebene müssen, wenn sie überzeugt sind, daß ein erstinstanzliches Urteil oder ein erstinstanzlicher Beschluß eines Volksgerichts derselben Ebene fehlerhaft ist, gemäß dem Verfahren über die Berufung Protest erheben.

$\S 18$

Wenn die Oberste Volksstaatsanwaltschaft bezüglich bereits rechtskräftiger Urteile und Beschlüsse von Volksgerichten jeder Ebene und wenn eine Volksstaatsanwaltschaft einer höheren Ebene bezüglich bereits rechtskräftiger Urteile und Beschlüsse von Volksgerichten einer niederen Ebene zu der Úberzeugung kommen, daß diese (Urteile/Beschlüsse) tatsächlich fehlerhaft sind, so haben sie gemäß dem Rechtsmittelverfahren Protest zu erheben.

Die (zuständige) Volksstaatsanwaltschaft hat zu im Rechtsmittelverfahren verhandelten Sachen Sitzungsvertreter zu entsenden.

$\$ 19$

Wenn die Volksstaatsanwaltschaft entdeckt, daß bei der Vollstreckung von Urteilen oder Beschlüssen in Strafsachen Gesetzesverletzungen vorgekommen sind, hat sie die Vollstrekkungsorgane anzuweisen, diese (Gesetzesverletzungen) abzustellen.

Wenn die Volksstaatsanwaltschaft entdeckt, daß in der Tätigkeit von Strafanstalten, Untersuchungshaftanstalten und Arbeitsumerziehungsanstalten Gesetzesverletzungen vorgekommen sind, hat sie das (mit der fraglichen Tätigkeit) befaßte Organ anzuweisen, diese (Gesetzesverletzung) abzustellen.

\section{DRITTES KAPITEL}

Die behördlichen Einrichtungen der Volksstaatsanwaltschaft und die Bestellung und Entlassung der Bediensteten

\section{$\int 20$}

Die Oberste Volksstaatsanwaltschaft richtet Dezernate für Strafsachen, Disziplinarrecht (Anm.: betreffend die VStA selbst), Vollzugsanstalten und Wirtschaftssachen ein; bei Bedarf kann sie weitere Behörden errichten.

Die örtlichen Volksstaatsanwaltschaften aller Ebenen sowie Sondervolksstaatsanwaltschaften können erforderliche Dienstbehörden einrichten. 


\section{$\ 21$}

Der Leitende Staatsanwalt der Obersten Volksstaatsanwaltschaft wird vom Nationalen Volkskongreß gewählt und abberufen.

Der Stellvertretende Leitende Staatsanwalt der Obersten Volksstaatsanwaltschaft, die Mitglieder ihres Staatsanwaltsausschusses und ihre Staatsanwälte werden vom Leitenden Staatsanwalt der Obersten Volksstaatsanwaltschaft nach Genehmigung des Ständigen Ausschusses des Nationalen Volkskongresses ernannt und entlassen.

$\int 22$

Die Leitenden Staatsanwälte und Leiter der Abteilungsvolksstaatsanwaltschaften der Provinzen, Autonomen Gebiete und regierungsunmittelbaren Städte werden von den Volksvertreterversammlungen der Provinzen, Autonomen Gebiete und regierungsunmittelbaren Städte gewählt und abberufen; die Stellvertretenden Leitenden Staatsanwälte, Mitglieder der Statsanwaltsausschüsse und Staatsanwälte werden vom Leitenden Volksstaatsanwalt der Provinzen, Autonomen Gebiete und regierungsunmittelbaren Städte nach Genehmigung des Ständigen Ausschusses der Volksvertreterversammlung derselben (jeweiligen) Ebene ernannt und entlassen.

Für die Ernennung und Entlassung der Leitenden Volksstaatsanwälte, Stellvertretenden Leitenden Volksstaatsanwälte und Mitglieder der Staatsanwaltsausschüsse in Provinzen, Autonomen Gebieten und regierungsunmittelbaren Städten ist dem Leitenden Obersten Volksstaatsanwalt Mitteilung zu machen, damit dieser die Genehmigung des Ständigen Ausschusses des Nationalen Volkskongresses (zu den Ernennungen und Entlassungen) einholt.

$\int 23$

Die Leitenden Volksstaatsanwälte der Autonomen Bezirke, provinzunmittelbaren Städte, der Kreise, Städte und stadtunmittelbaren Gebiete werden von der Volksvertreterversammlung derselben (jeweiligen) Ebene gewählt und abberufen; die Stellvertretenden Leitenden Staatsanwälte, Mitglieder der Staatsanwaltsausschüsse und Staatsanwälte werden vom Leitenden Volksstaatsanwalt der Autonomen Bezirke, provinzunmittelbaren Städte, der Kreise, Städte und stadtunmittelbaren Gebiete nach Genehmigung des Ständigen Ausschusses der Volksvertreterversammlung derselben (jeweiligen) Ebene ernannt und entlassen.

Für die Ernennung und Entlassung der Leitenden Volksstaatsanwälte, Stellvertretenden Leitenden Volksstaatsanwälte und Mitglieder der Staatsanwaltsausschüsse in Autonomen Bezirken, provinzunmittelbaren Städten, den Kreisen, Städten und stadtunmittelbaren Gebieten ist dem (jeweiligen) Leitenden Volksstaatsanwalt der Provinz, des Autonomen Gebiets oder der regierungsunmittelbaren Stadt Mitteilung zu machen, damit dieser die Genehmigung des Ständigen Ausschusses der Volksvertreterversammlung derselben (jeweiligen) Ebene (zu den Ernennungen und Entlassungen) einholt.

$\$ 24$

Die Leitenden Volksstaatsanwälte, Stellvertretenden Leitenden Volksstaatsanwälte, Mitglieder der Staatsanwaltsausschüsse und Staatsanwälte der von den Volksstaatsanwaltschaften der Provinz- und Kreisebenen in Bergwerksgebieten, landwirtschaftlichen Erschließungsgebieten und Forstgebieten eingerichteten (Volksstaatsanwaltschaften) werden sämtlich vom entsendenden Leitenden Volksstaatsanwalt nach Genehmigung des Ständigen Ausschusses der Volksvertreterversammlung derselben (jeweiligen) Ebene ernannt und entlassen.

$\S 25$

Die Amtszeit der Leitenden Volksstaatsanwälte aller Ebenen ist dieselbe wie die Amtsperiode der Volksvertreterversammlung derselben (jeweiligen) Ebene. 


\section{$\S 26$}

Die Ständigen Ausschüsse des Nationalen Volkskongresses und der Volksvertreterversammlungen der Provinzen, Autonomen Gebiete und regierungsunmittelbaren Städte können gemäß dem Vorschlag des Leitenden Volksstaatsanwalts derselben (jeweiligen) Ebene Leitende Volksstaatsanwälte, Stellvertretende Leitende Volksstaatsanwälte und Mitglieder der Staatsanwaltsausschüsse der (jeweils) niederen Ebenen auswechseln.

$\int 27$

Die Volksstaatsanwaltschaften aller Ebenen bestellen jeweils mehrere Hilfsstaatsanwälte und Sekretäre. Nach Genehmigung durch den Leitenden Staatsanwalt können die Hilfsstaatsanwälte stellvertretend Aufgaben der Staatsanwälte übernehmen. Die Sekretäre erledigen die Schriftführung betreffend die Fälle (der jeweiligen VStA) sowie die dazugehörigen Angelegenheiten.

Die Hilfsstaatsanwälte und Sekretäre werden vom Leitenden Volksstaatsanwalt einer jeden Ebene ernannt und entlassen.

Die Volksstaatsanwaltschaften jeder Ebene können gemäß den Erfordernissen (ihrer Tätigkeit) Justizwachtmeister (Anm.: wörtlich , Justizpolizei“) bestellen.

$\$ 28$

Die Personalstruktur der Volksstaatsanwaltschaften aller Ebenen wird von der Obersten Volksstaatsanwaltschaft anderweitig geregelt.

$$
\text { (übersetzt von Wolfgang Keßler) }
$$

